PROCEEDINGS OF THE

AMERICAN MATHEMATICAL SOCIETY

Volume 125, Number 11, November 1997, Pages 3433-3438

S 0002-9939(97)04147-6

\title{
SUPERRIGID SUBGROUPS OF SOLVABLE LIE GROUPS
}

\author{
DAVE WITTE
}

(Communicated by Roe Goodman)

\begin{abstract}
Let $\Gamma$ be a discrete subgroup of a simply connected, solvable Lie group $G$, such that $\operatorname{Ad}_{G} \Gamma$ has the same Zariski closure as $\operatorname{Ad} G$. If $\alpha: \Gamma \rightarrow$ $\mathrm{GL}_{n}(\mathbb{R})$ is any finite-dimensional representation of $\Gamma$, we show that $\alpha$ virtually extends to a continuous representation $\sigma$ of $G$. Furthermore, the image of $\sigma$ is contained in the Zariski closure of the image of $\alpha$. When $\Gamma$ is not discrete, the same conclusions are true if we make the additional assumption that the closure of $[\Gamma, \Gamma]$ is a finite-index subgroup of $[G, G] \cap \Gamma$ (and $\Gamma$ is closed and $\alpha$ is continuous).
\end{abstract}

\section{INTRODUCTION}

The Margulis Superrigidity Theorem [4, Thm. VII.5.9, p. 230] concerns lattices in semisimple Lie groups. The author $[7]$ (see $[8, \S 6]$ for errata) proved an analogue of this fundamental theorem for many lattices in Lie groups that are not semisimple, with most of his attention devoted to solvable groups (see 3.2). We now prove that, in the case of solvable groups, there is no need to restrict attention to latticesexactly the same result holds for any discrete subgroup.

1.1. Definition. We say that a representation $\alpha: \Gamma \rightarrow \mathrm{GL}_{n}(\mathbb{R})$ virtually extends to a representation of $G$ if the restriction of $\alpha$ to some finite-index subgroup of $\Gamma$ extends to a representation of $G$.

1.2. Definition. Let us say that a closed subgroup $\Gamma$ of a simply connected, solvable Lie group $G$ is superrigid in $G$ if every finite-dimensional representation $\alpha: \Gamma \rightarrow \mathrm{GL}_{n}(\mathbb{R})$ virtually extends to a representation $\sigma$ of $G$, such that the image $G^{\sigma}$ is contained in the Zariski closure of $\Gamma^{\alpha}$.

1.3. Remark. By considering induced representations, we see that every finite-index subgroup of a superrigid subgroup is superrigid.

2.2'. Definition. If $X$ is a subgroup of a Lie group $G$, let $\overline{\operatorname{Ad}_{G} X}$ denote the (almost) Zariski closure of $\operatorname{Ad}_{G} X$ in the real algebraic group Aut $\mathcal{G}$, where $\mathcal{G}$ is the Lie algebra of $G$.

1.4. Theorem (see 3.5). Let $\Gamma$ be a discrete subgroup of a simply connected, solvable Lie group $G$, such that $\overline{\operatorname{Ad}_{G} \Gamma}=\overline{\operatorname{Ad} G}$. Then $\Gamma$ is superrigid in $G$.

For subgroups that are not discrete, an additional hypothesis is required.

Received by the editors June 21, 1996.

1991 Mathematics Subject Classification. Primary 22E40; Secondary 22E25, 22E27, 22G05.

(C)1997 American Mathematical Society 
1.5. Theorem (see 3.6). Let $\Gamma$ be a closed subgroup of a simply connected, solvable Lie group $G$, such that $\overline{\operatorname{Ad}_{G} \Gamma}=\overline{\operatorname{Ad} G}$. If the closure of $[\Gamma, \Gamma]$ is a finite-index subgroup of $[G, G] \cap \Gamma$, then $\Gamma$ is superrigid in $G$.

If $\Gamma$ is superrigid in $G$, then $\Gamma$ is also superrigid in any semidirect product $A \rtimes G$. The following theorem shows that combining this observation with Theorem 1.5 is (virtually) the only way to construct a superrigid subgroup.

3.8. Theorem. A closed subgroup $\Gamma$ of a simply connected, solvable Lie group $G$ is superrigid iff $\Gamma$ has a finite-index subgroup $\Gamma^{\prime}$, such that

(1) there is a semidirect-product decomposition $G=A \rtimes B$, with $\Gamma^{\prime} \subset B$ and $\overline{\operatorname{Ad}_{B} \Gamma^{\prime}}=\overline{\operatorname{Ad} B}$, and

(2) the closure of $\left[\Gamma^{\prime}, \Gamma^{\prime}\right]$ is a finite-index subgroup of $[B, B] \cap \Gamma^{\prime}$.

If $\Gamma$ is discrete, then Hypothesis (2) of the theorem follows from (1) (see 2.5(1)), so we have the following corollary.

1.6. Corollary. A discrete subgroup $\Gamma$ of a simply connected, solvable Lie group $G$ is superrigid iff there is a semidirect-product decomposition $G=A \rtimes B$, such that $B$ contains a finite-index subgroup $\Gamma^{\prime}$ of $\Gamma$, and $\overline{\operatorname{Ad}_{B} \Gamma^{\prime}}=\overline{\operatorname{Ad} B}$.

\section{Preliminaries}

2.1. Definition ([7, Defn. 3.2]). A subgroup $A$ of $\mathrm{GL}_{n}(\mathbb{R})$ is said to be almost Zariski closed if there is a Zariski closed subgroup $B$ of $\mathrm{GL}_{n}(\mathbb{R})$, such that $B^{\circ} \subset$ $A \subset B$, where $B^{\circ}$ is the identity component of $B$ in the topology of $\mathrm{GL}_{n}(\mathbb{R})$ as a $C^{\infty}$ manifold (not the Zariski topology). There is little difference between being Zariski closed and almost-Zariski closed, because $B^{\circ}$ always has finite index in $B$.

2.2. Definition ([7, Defn. 3.6]). The almost-Zariski closure $\bar{A}$ of a subgroup $A$ of $\mathrm{GL}_{n}(\mathbb{R})$ is the unique smallest almost-Zariski closed subgroup that contains $A$. In particular, if $A$ is a subgroup of a Lie group $G$, we use $\overline{\operatorname{Ad}_{G} A}$ to denote the almostZariski closure of $\operatorname{Ad}_{G} A$ in the real algebraic group $\operatorname{Aut}(\mathcal{G})$, where $\mathcal{G}$ is the Lie algebra of $G$.

2.3. Definition ([7, §5], cf. $[2$, p. 6$])$. Let $\Gamma$ be a closed subgroup of a Lie group $G$. A syndetic hull of $\Gamma$ is a connected subgroup $B$ that contains $\Gamma$, such that $B / \Gamma$ is compact.

2.4. Proposition ([7, Prop. 5.9, p. 168]). Let $\Gamma$ be a closed subgroup of a connected, solvable Lie group $G$, such that $\overline{\operatorname{Ad}_{G} \Gamma}$ contains a maximal compact torus of $\overline{\operatorname{Ad} G}$. Then $\Gamma$ has a syndetic hull in $G$.

2.5. Lemma ([7, Prop. 5.10 and Cor. 5.11, p. 168]). Suppose $\Gamma$ is a closed subgroup of a simply connected, solvable Lie group $G$, such that $\overline{\operatorname{Ad}_{G} \Gamma}=\overline{\operatorname{Ad} G}$. Then the closure of $[\Gamma, \Gamma]$ contains a cocompact subgroup of $[G, G] \cap \Gamma$. Furthermore:

(1) If $\Gamma$ is discrete, then $[\Gamma, \Gamma]$ is a finite-index subgroup of $[G, G] \cap \Gamma$.

(2) If $\Gamma$ is connected, then $[\Gamma, \Gamma]=[G, G]$.

2.6. Definition. A real-valued function $f$ on a subset $G$ of $\mathrm{GL}_{m}(\mathbb{R})$ is regular if there are polynomials $p$ and $q$ in $n^{2}+1$ variables, such that, for all $g \in G$, we have

$$
q\left(g_{i j}, \operatorname{det} g^{-1}\right) \neq 0 \quad \text { and } \quad f(g)=\frac{p\left(g_{i j}, \operatorname{det} g^{-1}\right)}{q\left(g_{i j}, \operatorname{det} g^{-1}\right)} .
$$


A function $f: G \rightarrow \mathrm{GL}_{n}(\mathbb{R})$ is regular if each matrix entry of $f(g)$ is a regular function of $g$.

2.7. Lemma ([7, Prop. 3.16, p. 158]). Suppose $G$ is an almost-Zariski closed subgroup of $\mathrm{GL}_{m}(\mathbb{R})$, and $\phi: G \rightarrow \mathrm{GL}_{n}(\mathbb{R})$ is a regular homomorphism. Then $G^{\phi}$ is almost-Zariski closed.

2.8. Lemma ([7, Lem. 3.17, p. 159]). Suppose $A$ and $B$ are almost-Zariski closed subgroups of $\mathrm{GL}_{n}(\mathbb{R})$, such that $A B$ is a subgroup. Then $A B$ is almost-Zariski closed.

2.9. Lemma ([7, Cor. 2.19 , p. 154$])$. If $N$ is a connected, normal subgroup of a simply connected, solvable Lie group $G$, then $G / N$ is simply connected. Therefore, $G / N$ has no nontrivial compact subgroups.

2.10. Lemma (Ado-Iwasawa). If $G$ is a simply connected, solvable Lie group, then $G$ has a faithful, finite-dimensional representation $\alpha$, such that $G^{\alpha}$ is closed.

Proof. The usual statement of the Ado-Iwasawa Theorem [3, Thm. XVIII.3.1, p. 219] states that $G$ has a faithful, finite-dimensional representation $\sigma$, such that $(\operatorname{nil} G)^{\sigma}$ is unipotent. Hence (nil $\left.G\right)^{\sigma}$ is closed (see 2.13). For the same reason, because $G / \operatorname{nil} G$ is abelian, there is a faithful representation $\tau$ of $G / \operatorname{nil} G$, whose image is closed. The direct sum of $\sigma$ and $\tau$ is the desired representation $\alpha$.

2.11. Lemma ([7, Lem. 5.6, p. 166]). Let $G$ be a connected, solvable Lie group, and let $A$ be an almost-Zariski closed subgroup of $\mathrm{GL}_{n}(\mathbb{R})$. If $\rho: G \rightarrow \mathrm{GL}_{n}(\mathbb{R})$ is a continuous homomorphism, such that $A$ contains a maximal compact torus of $\overline{G^{\rho}}$, then the inverse image $\rho^{-1}(A)$ is a connected subgroup of $G$.

2.12. Lemma. Let $G$ and $H$ be simply connected, solvable Lie groups. If $\sigma: G \rightarrow$ $H$ is a continuous homomorphism, then the kernel of $\sigma$ is connected.

Proof. Let $K=\operatorname{ker} \sigma$. Because every connected subgroup of a simply connected, solvable Lie group is simply connected [3, Thm. XII.2.2, p. 137], we know $G^{\sigma}$ is simply connected. In other words, $G / K$ is simply connected. Because $G$ is connected and solvable, this implies that $K$ is connected [7, Lem. 2.17, p. 154].

2.13. Lemma ([7, Lem. 3.20, p. 159]). Every connected, unipotent Lie subgroup of $\mathrm{GL}_{n}(\mathbb{R})$ is Zariski closed.

2.14. Lemma (cf. [1, Cor. I.5.3.7, p. 47]). Let $A$ be a connected, nilpotent, normal subgroup of a Lie group $G$. Then $\overline{\operatorname{Ad}}_{G} \operatorname{nil} G$ is unipotent.

The following well-known result is a consequence of the fact that maximal compact tori are conjugate (see [5, Prop. 3.10(2), p. 126] and [9, Cor. 102.9.1, p. 293]).

2.15. Lemma. Let $K$ and $G$ be almost-Zariski closed, connected subgroups of $\mathrm{GL}_{n}(\mathbb{R})$. If $K$ is a normal subgroup of $G$, then every maximal compact torus of $G$ contains a maximal compact torus of $K$.

\section{The THEOREMS AND THEIR PROOFs}

The following are two versions of the main theorem of [7]. In this section, we eliminate the cocompactness assumption in these results (see 3.4) and prove a converse (see 3.8). 
3.1. Theorem ([7, Cor. 6.6, p. 175]). Let $\Gamma$ be a closed, cocompact subgroup of a simply connected, solvable Lie group $G$, and assume $\overline{\operatorname{Ad}_{G} \Gamma}=\overline{\operatorname{Ad} G}$. Suppose $\alpha: \Gamma \rightarrow \mathrm{GL}_{n}(\mathbb{R})$ is a representation of $\Gamma$. If $\overline{\Gamma^{\alpha}}$ and $Z\left(\overline{\Gamma^{\alpha}}\right)$ are connected, and $([G, G] \cap \Gamma)^{\alpha}$ is unipotent, then $\alpha$ extends to a homomorphism from $G$ to $\overline{\Gamma^{\alpha}}$.

3.2. Corollary ([7, Cor. 6.8 , p. 176$])$. Let $\Gamma$ be a lattice subgroup of a simply connected, solvable Lie group $G$, such that $\overline{\operatorname{Ad}_{G} \Gamma}=\overline{\operatorname{Ad} G}$. Then $\Gamma$ is superrigid in $G$.

3.3. Remark. If one is interested only in 1.4, not the more precise results such as Theorem 3.4 and its corollaries, a direct proof can be obtained from the proof of 3.4 by appealing to Corollary 3.2 instead of Theorem 3.1. A short, fairly easy proof of Corollary 3.2 appears in $[8, \S 2]$.

3.4. Theorem. Let $\Gamma$ be a closed subgroup of a simply connected, solvable Lie group $G$, and assume $\overline{\operatorname{Ad}_{G} \Gamma}=\overline{\operatorname{Ad} G}$. Suppose $\alpha: \Gamma \rightarrow \mathrm{GL}_{n}(\mathbb{R})$ is a representation of $\Gamma$. If $\overline{\Gamma^{\alpha}}$ and $Z\left(\overline{\Gamma^{\alpha}}\right)$ are connected, and $([G, G] \cap \Gamma)^{\alpha}$ is unipotent, then $\alpha$ extends to a homomorphism from $G$ to $\overline{\Gamma^{\alpha}}$.

Proof. Because $\overline{\operatorname{Ad}_{G} \Gamma}=\overline{\operatorname{Ad} G}$, we know that $\Gamma$ has a syndetic hull $B$ in $G$ (see 2.4). Then $\alpha$ extends to a homomorphism $\sigma: B \rightarrow \overline{\Gamma^{\alpha}}$ (see 3.1). From Lemma 2.5(2), we see that $([G, G] \cap B)^{\sigma}=[B, B]^{\sigma} \subset\left[\overline{\Gamma^{\alpha}}, \overline{\Gamma^{\alpha}}\right]$ is unipotent, so there is no harm in replacing $\Gamma$ with $B$. Thus, we may assume $\Gamma$ is connected.

We may assume $G \subset \mathrm{GL}_{n}(\mathbb{R})$ (see 2.10), so there is no harm in speaking of the almost-Zariski closure of $G$ or of its subgroups. Let $\pi: \bar{G} \times \overline{\Gamma^{\alpha}} \rightarrow \bar{G}$ be the projection onto the first factor.

Let $X \subset \Gamma \times \overline{\Gamma^{\alpha}}$ be the graph of $\alpha$. It suffices to find a closed subgroup $Y$ of $G \times \overline{\Gamma^{\alpha}}$ such that $X \subset Y, Y \cap \overline{\Gamma^{\alpha}}=e$, and $Y^{\pi}=G$. For then $Y$ is the graph of a homomorphism $G \rightarrow \overline{\Gamma^{\alpha}}$ that extends $\alpha$, as desired.

Because $\bar{X}^{\pi} \supset X^{\pi}=\Gamma$, and $\operatorname{Ad}_{G} \bar{X}^{\pi}$ is almost-Zariski closed (see 2.7), we have $\operatorname{Ad}_{G} \bar{X}^{\pi} \supset \overline{\operatorname{Ad}_{G} \Gamma}=\overline{\operatorname{Ad} G}$, so $G \subset \bar{X}^{\pi} Z(\bar{G})$. Thus, letting $\hat{X}=\bar{X} Z(\bar{G})^{\circ}$, we have $G \subset \hat{X}^{\pi}$.

Let $Y$ be a connected subgroup of $\hat{X}$ that contains $X$ and is maximal, subject to the conditions that $Y \cap \overline{\Gamma^{\alpha}}=e$ and $Y^{\pi} \subset G$. We claim that $Y^{\pi}=G$ (which will complete the proof). If not, then, because $G \subset \hat{X}^{\pi}$, there is a one-parameter subgroup $A$ of $\hat{X}$ such that $A^{\pi}$ is contained in $G$, but is not contained in $Y^{\pi}$. From Lemma 2.5(2), we know that $[\bar{X}, \bar{X}]=[X, X] \subset X \subset Y$, so $Y$ is normal in $\hat{X}$; hence $A Y$ is a subgroup. Furthermore, because $G$ is simply connected and $Y^{\pi}$ is connected, we know that $G / Y^{\pi}$ has no nontrivial compact subgroups (see 2.9). Because $A^{\pi} \not \subset Y^{\pi}$, this implies that $a^{\pi} \notin Y^{\pi}$, for all $a \in A-\{e\}$; therefore $(A Y) \cap \overline{\Gamma^{\alpha}}=Y \cap \overline{\Gamma^{\alpha}}$ is trivial. This contradicts the maximality of $Y$.

3.5. Corollary (cf. pf. of $[7$, Cor. 6.7, p. 176]). Let $\Gamma$ be a discrete subgroup of a simply connected, solvable Lie group $G$, and assume $\overline{\operatorname{Ad}_{G} \Gamma}=\overline{\operatorname{Ad} G}$. Suppose $\alpha: \Gamma \rightarrow \mathrm{GL}_{n}(\mathbb{R})$ is a homomorphism, such that $\overline{\Gamma^{\alpha}}$ is connected. Then there is a homomorphism $\sigma: G \rightarrow \overline{\Gamma^{\alpha}}$ and a finite subgroup $Z_{0}$ of $Z\left(\overline{\Gamma^{\alpha}}\right)$ such that $\gamma^{\sigma} \in \gamma^{\alpha} Z_{0}$, for every $\gamma \in \Gamma$. In particular, $\sigma$ virtually extends $\alpha$.

To obtain the same conclusion for subgroups that are not discrete, we add the conclusion of Lemma 2.5(1) as a hypothesis. 
3.6. Corollary (cf. pf. of $[7$, Cor. 6.7, p. 176]). Let $\Gamma$ be a closed subgroup of a simply connected, solvable Lie group $G$, such that the closure of $[\Gamma, \Gamma]$ is a finiteindex subgroup of $[G, G] \cap \Gamma$, and assume $\overline{\operatorname{Ad}_{G} \Gamma}=\overline{\operatorname{Ad} G}$. Suppose $\alpha: \Gamma \rightarrow \mathrm{GL}_{n}(\mathbb{R})$ is a homomorphism, such that $\overline{\Gamma^{\alpha}}$ is connected. Then there is a homomorphism $\sigma: G \rightarrow \overline{\Gamma^{\alpha}}$ and a finite subgroup $Z_{0}$ of $Z\left(\overline{\Gamma^{\alpha}}\right)$ such that $\gamma^{\sigma} \in \gamma^{\alpha} Z_{0}$, for every $\gamma \in \Gamma$. In particular, $\sigma$ virtually extends $\alpha$.

Without the additional hypothesis, we obtain only the following weaker result, by using the fact that, although the closure of $[\Gamma, \Gamma]$ may not have finite index in $[G, G] \cap \Gamma$, it is cocompact (see 2.5).

3.7. Corollary (cf. pf. of $[7$, Cor. 6.7, p. 176]). Let $\Gamma$ be a closed subgroup of a simply connected, solvable Lie group $G$, and assume $\overline{\operatorname{Ad}_{G} \Gamma}=\overline{\operatorname{Ad} G}$. Suppose $\alpha: \Gamma \rightarrow \mathrm{GL}_{n}(\mathbb{R})$ is a homomorphism, such that $\overline{\Gamma^{\alpha}}$ is connected. Then there is a homomorphism $\sigma: G \rightarrow \overline{\Gamma^{\alpha}}$ and a compact subgroup $Z_{0}$ of $Z\left(\overline{\Gamma^{\alpha}}\right)$ such that $\gamma^{\sigma} \in \gamma^{\alpha} Z_{0}$, for every $\gamma \in \Gamma$.

3.8. Theorem. A closed subgroup $\Gamma$ of a simply connected, solvable Lie group $G$ is superrigid iff $\Gamma$ has a finite-index subgroup $\Gamma^{\prime}$, such that

(1) there is a semidirect-product decomposition $G=A \rtimes B$, with $\Gamma^{\prime} \subset B$ and $\overline{\operatorname{Ad}_{B} \Gamma^{\prime}}=\overline{\operatorname{Ad} B}$, and

(2) the closure of $\left[\Gamma^{\prime}, \Gamma^{\prime}\right]$ is a finite-index subgroup of $[B, B] \cap \Gamma^{\prime}$.

Proof. $(\Rightarrow)(1)$ There is a faithful representation $\alpha: G \rightarrow \mathrm{GL}_{n}(\mathbb{R})$, such that $G^{\alpha}$ is closed (see 2.10). (So $\Gamma^{\alpha}$ is also closed.) Because $\Gamma$ is superrigid, we know that, after replacing $\Gamma$ by a finite-index subgroup, the homomorphism $\left.\alpha\right|_{\Gamma}$ extends to a homomorphism $\sigma: G \rightarrow \overline{\Gamma^{\alpha}}$. Because $G$ is simply connected, we may lift $\sigma$ to a homomorphism $\tilde{\sigma}: G \rightarrow \widetilde{\widetilde{\Gamma^{\alpha}}}$, where $\widetilde{\overline{\Gamma^{\alpha}}}$ is the universal cover of $\overline{\Gamma^{\alpha}}$. By combining Lemma 2.7 with the fact that $\overline{\Gamma^{\alpha}}=\overline{G^{\sigma}}$, we see that

$$
\overline{\operatorname{Ad}_{G^{\sigma}} \Gamma^{\alpha}}=\operatorname{Ad}_{G^{\sigma}} \overline{\Gamma^{\alpha}}=\operatorname{Ad}_{G^{\sigma}} \overline{G^{\sigma}}=\overline{\operatorname{Ad} G^{\sigma}} .
$$

Therefore, by passing to the covering group $G^{\widetilde{\sigma}}$, we have $\overline{\operatorname{Ad}_{G^{\tilde{\sigma}}} \Gamma^{\tilde{\sigma}}}=\overline{\operatorname{Ad} G^{\tilde{\sigma}}}$. Thus, letting $K=\operatorname{ker} \widetilde{\sigma}$, we see that $\overline{\operatorname{Ad}_{G} K \Gamma}=\overline{\operatorname{Ad} G}$. Furthermore, $K$ is connected (see 2.12); we have $K \cap \Gamma=e$, because $\left.\sigma\right|_{\Gamma}=\alpha$ is faithful, and we know that $K \Gamma$ is closed, because $\Gamma^{\alpha}$ is closed. So Lemma 3.9 below implies that there is a semidirect-product decomposition $G=A \rtimes B$ with $K \subset A$ and $\Gamma \subset B$. Because $\overline{\operatorname{Ad}_{G} A \Gamma} \supset \overline{\operatorname{Ad}_{G} K \Gamma}=\overline{\operatorname{Ad} G}$, we see that $\overline{\operatorname{Ad}_{B} \Gamma}=\overline{\operatorname{Ad} B}$.

(2) Because $\Gamma^{\prime}$ is a finite-index subgroup of $\Gamma$, we know that $\Gamma^{\prime}$ is a superrigid subgroup of $G$ (see 1.3). Thus, by replacing $\Gamma$ with $\Gamma^{\prime}$, we may assume $\Gamma \subset B$. Let $D$ be the closure of $[\Gamma, \Gamma]$. Because $\Gamma / D$ is abelian, there is a faithful homomorphism $\alpha: \Gamma / D \rightarrow T$, for some compact torus $T \subset \mathrm{GL}_{n}(\mathbb{R})$. We may think of $\alpha$ as a representation of $\Gamma$ that is trivial on $D$. Then, because $\Gamma$ is superrigid, we know that $\alpha$ virtually extends to a homomorphism $\sigma: G \rightarrow T$. Of course $[G, G]^{\sigma}=e$, because $T$ is abelian. Thus, because $\alpha$ agrees with $\sigma$ on a finite-index subgroup of $\Gamma$, we conclude that $D$, the kernel of $\alpha$, contains a finite-index subgroup of $[G, G] \cap \Gamma$.

3.9. Lemma. Let $K$ and $\Gamma$ be closed subgroups of a simply connected, solvable Lie group $G$, such that $K$ is connected and normal in $G, K \cap \Gamma=e, K \Gamma$ is closed, and $\overline{\operatorname{Ad}_{G} K \Gamma}=\overline{\operatorname{Ad} G}$. Then there is a semidirect-product decomposition $G=A \rtimes B$ with $K \subset A$ and $\Gamma \subset B$. 
Proof. By induction on the derived length of $K$, we may assume $K$ is abelian, so $\operatorname{Ad}_{G} K$ is unipotent (see 2.14). Then, because $\overline{\operatorname{Ad}_{G} K \Gamma}=\overline{\operatorname{Ad} G}$, we know that $\overline{\operatorname{Ad}_{G} \Gamma}$ contains a maximal compact torus of $\overline{\operatorname{Ad} G}$. So Lemma 2.4 implies that $\Gamma$ has a syndetic hull $X$ in $G$. Thus, by replacing $\Gamma$ with $X$, we may assume $\Gamma$ is connected.

Let $H=\left\{g \in G \mid \operatorname{Ad}_{G} g \in \overline{\operatorname{Ad}_{G} \Gamma}\right\}$. Because $\overline{\operatorname{Ad}_{G} \Gamma}$ contains a maximal compact torus of $\overline{\operatorname{Ad} G}$, we know that $H$ is connected (see 2.11). For much the same reason, $K \cap H$ is connected (see 2.15).

We claim that $G=K H$. Given $g \in G$, because $\overline{\operatorname{Ad} G}=\overline{\operatorname{Ad}_{G} K \Gamma}$, we see, from Lemma 2.8, that $\operatorname{Ad}_{G} g=k^{\prime} h^{\prime}$, for some $k^{\prime} \in \overline{\operatorname{Ad}_{G} K}$ and $h^{\prime} \in \overline{\operatorname{Ad}_{G} \Gamma}$. But, because $\operatorname{Ad}_{G} K$ is unipotent, we have $\operatorname{Ad}_{G} K=\overline{\operatorname{Ad}_{G} K}$ (see 2.13), so there is some $k \in K$ with $\operatorname{Ad}_{G} k=k^{\prime}$. Then $\operatorname{Ad}_{G}\left(k^{-1} g\right)=h^{\prime} \in \overline{\operatorname{Ad}_{G} \Gamma}$, so, from the definition of $H$, we have $k^{-1} g \in H$.

From the preceding paragraph, we see that, by replacing $K$ with $K \cap H$, we may assume $G=H$. Therefore, $\overline{\operatorname{Ad}_{G} \Gamma}=\overline{\operatorname{Ad} G}$, so $[G, G] \subset \Gamma$ (see 2.5(2)). Thus, it is easy to find a subgroup $B \supset \Gamma$ with $G=K \ltimes B$. Since $K$ is normal in $G$, we see that $G$ is the direct product $K \times B$.

\section{ACKNOWLEDGMEnT}

I am grateful to A. Magid for asking the astute question that directed me to this extension of my earlier work. This research was partially supported by a grant from the National Science Foundation.

\section{REFERENCES}

[1] N. Bourbaki, "Lie Groups and Lie Algebras, Part I," Addison-Wesley, Reading, MA, 1975.

[2] D. Fried and W. Goldman, Three-dimensional affine crystallographic groups, Adv. Math 47 (1983) 1-49. MR 84d:20047

[3] G. Hochschild, "The Structure of Lie Groups," Holden-Day, San Francisco, 1965. MR 34:7696

[4] G. A. Margulis, "Discrete Subgroups of Semisimple Lie Groups," Springer-Verlag, Berlin/New York, 1991. MR 92h:22021

[5] V. Platonov, A. Rapinchuk, "Algebraic Groups and Number Theory," Academic Press, Boston, 1994.

[6] M. S. Raghunathan, "Discrete Subgroups of Lie Groups," Springer-Verlag, Berlin/New York, 1972. MR 58:22394a

[7] D. Witte, Superrigidity of lattices in solvable Lie groups, Invent. Math. 122 (1995) 147-193. MR 96k:22024

[8] D. Witte, Archimedean superrigidity of solvable S-arithmetic groups, J. Algebra 187 (1997) $268-288$.

[9] D. P. Želobenko, "Compact Lie Groups and their Representations", American Mathematical Society, Providence, R. I., 1973. MR 57:12776b

Department of Mathematics, Oklahoma State University, Stillwater, Oklahoma 74078

E-mail address: dwitte@math.okstate.edu 